\title{
Clear speech perception in patients with auditory neuropathy spectrum disorder
}

\author{
Original \\ Article \\ Salwa Mourad Abd-Elmawgoud', Somaia M. Tawfik', Mohammed A. Abd Al- \\ Ghaffar $^{2}$
}

Audiology Unit, Otorhinolaryngology Department, ' Ain Shams University, ${ }^{2}$ Sohag University

\begin{abstract}
Introduction: One of the main characteristic of auditory neuropathy spectrum disorder (ANSD) is a significantly impaired temporal processing ability with subsequent difficulty in speech understanding particularly in noise. Enhancing temporal properties in clear speech can be beneficial in enhancing speech intelligibility in ANSD patients. Clear speech was developed in English language.

Objective: To develop and standardize Arabic clear speech materials and to evaluate performance of patients with ANSD and sensory neural hearing loss (SNHL) in developed Arabic clear speech.

Patients and Methods: The present study was conducted in three phases. Phase one; developing and recording of clear and conversational Arabic speech materials. Phase two; establishing norms in the developed materials by evaluation of 30 adult normal subjects. Phase three; comprised evaluation of performance of 30 adult SNHL patients and 40 adult patients with ANSD. Patients were tested in quiet as well as in noisy listening conditions.

Results: Clear speech advantage in ANSD patients was $16.7 \%$ over conversational speech, while it was $5 \%$ in patients with SNHL. All patients with ANSD performed more poorly in noise at all signal to noise ratios (SNRs).

Conclusion: The advantage of clear speech over conversational speech was more evident in ANSD patients than SNHL group. ANSD patients derive this advantage from enhancement of temporal properties in clear speech most likely. This study suggested using clear speech in remediation of patients with ANSD in order to improve their ability to communicate.
\end{abstract}

Key Words: Auditory neuropathy spectrum disorder, clear speech, speech perception, temporal processing

Corresponding Author: Salwa Mourad Abd-Elmawgoud, M.D., Audiology Unit, Otorhinolaryngology Department, Sohag University, Sohag, Egypt, Tel.: 01224688699, E-mail: salwa_mawgoud@yahoo.com

ISSN: 2090-0740, March 2018 Vol.19, No.1

\section{INTRODUCTION}

Auditory neuropathy spectrum disorder (ANSD) or Auditory Dys-synchrony is a relatively recent diagnosis in the field of audiology. It was first described by Soliman (1987) as a low frequency syndrome. The term 'Auditory Neuropathy' was coined by Starr and colleagues in 1996 (Berlin et al., 2002; Rapin and Gravel, 2003). One main characteristic of ANSD is the disrupted auditory nerve activity with concurrently normal or nearly normal cochlear amplification function (Starr et al., 1996; Hood et al., 2003 and Rosamaria et al., 2008). The other main characteristic of ANSD is a significantly impaired capacity for temporal processing and difficulty in speech understanding, particularly in noise, that is disproportionate to the degree of hearing loss measured by pure-tone audiometry (Zeng et al., 2005). The prevalence of ANSD has been estimated to affect about $10 \%$ of infants who failed hearing screening (Gunay et al., 2008, Bielecki et al., 2012). However, the prevalence in adults remains questionable with the reported rates of occurrence ranging from as low as $0.5 \%$ of the hearing impaired population to as high as $15 \%$ (Simmons and McCreery, 2007). The specific risk factors for ANSD have not been fully revealed yet. While a significant number of patients have no risk factors, others suggest a history of risk factors as prematurity, low birth weight, anoxia, and hyperbilirubinemia. Genetics also play an important role. ANSD appears to follow both recessive and dominant inheritance patterns (Hood et al., 2002; Beutner, 2007 and Xoinis et al., 2007). Rance et al. (2004) reported that there are many pathologies that could produce the ANSD result profile. Some of them include insult specific to the cochlear inner hair cells (IHCs), abnormality of inner hair cells/ auditory nerve fiber synapse, spiral ganglion cell disorder, depleted neuronal populations in the auditory brain stem, and demyelination of the auditory nerve. Unfortunately, the exact site of lesion(s) remains undetermined as there are no procedures presently available to assess the status of inner hair cells or of the synapse between the inner hair cells and auditory nerve fibers (Berlin et al., 2010, Nikolopoulos, 2014). There is no consensus on a specific therapeutic approach. There are several management 
options of ANSD including the use of hearing aids, some types of communication methods (i.e. sign language-cued speech-, or auditory verbal therapy), frequency modulated (FM) auditory trainers, or cochlear implants (Spirakis, 2012). According to Petros et al. (2008) the important feature of ANSD is the speech recognition deficit. One effective means of improving speech intelligibility is to speak clearly. The higher intelligibility in clear speech than in conversational speech is likely a result of acoustic and phonetic differences between these two styles of speech (Krause and Braida, 2002, and Liu et al., 2004). The benefit has been demonstrated in diverse populations including those with learning disabilities, auditory neuropathy, and cochlear implants (Liu et al., 2004). Because a temporal processing deficit is a hallmark of ANSD (Zeng, et al., 2005), temporal modification of speech might be beneficial for better speech understanding in ANSD (Hassan, 2008). Zeng et al. (2005) concluded that the enhanced temporal properties in clear speech may be, especially, beneficial to individuals with ANSD. Clear speech was developed in English language and several studies showed beneficial improvement in speech intelligibility of ANSD patients using clear speech (Liu et al., 2004 and Zeng, et al., 2005).

\section{Aims of the study:}

To develop and standardize Arabic clear speech materials, to evaluate the performance of ANSD patients on Arabic clear speech, and lastly, to compare the performance of patients with SNHL and ANSD on Arabic clear speech.

\section{METHODOLOGY:}

\section{Subjects:}

The present study consisted of two groups: Control group: including 30 subjects with age range from 20-50 years, no history of hearing loss, ear disease, trauma, ototoxicdrugintakeorearoperations. Normalhearing sensitivity did not exceeding $25 \mathrm{~dB}$ HL in the frequencies from $250 \mathrm{~Hz}$ to $8000 \mathrm{~Hz}$ by air conduction. Excellent word discrimination score. Normal middle ear functions as evidenced by ear examination, tympanometry and acoustic reflex thresholds. Study group : divided into two subgroups ; the first subgroup included 40 patients who were diagnosed as Auditory neuropathy spectrum disorder by pure tone, speech tests, OAE and ABR. Age range from 20-50 years. The degree of hearing loss ranged from mild to moderately severe degree of HTL. The hearing loss was bilateral and symmetrical. The patients did not use any management method previously. All patients were neurologically free.

The second subgroup included 30 patients with sensorineural hearing loss (SNHL). Age ranged from 20-50 years. The degree of hearing loss ranged from mild to moderately severe degree of HTL.

\section{Speech material:}

\section{Development of the stimulus:}

The stimuli used included speech sentences recorded in clear and conversational speech styles. These sentences were collected from the Arabic Language book for $6^{\text {th }}$ primary school and from encyclopedia on line (www. wikipedia.org). The 144 sentences were separated into 18 lists, each list contains 8 sentences and 25 key words in each list (see the appendix). The first sentence in each list has four key words, and the remaining sentences have three.

\section{Criteria of clear speech sentences:}

Slower speaking rate, the duration was double that of conversational sentences. More and longerpauses. Increased energy in the 1000-3000 Hz range. Targeted vowel formants. Increased consonant intensity compared to adjacent vowels (more stress on consonant). Expanded voice pitch range.

\section{Recording of the stimulus:}

Sentences were recorded in the Acoustic Studio (ElHakem Studio). Male adult professional talker recorded these lists with a sampling rate of $16 \mathrm{kHz}$ in a soundtreated room. Using a $150-\mathrm{Hz}$ high pass filter to remove occasional breathing noise. A $1000-\mathrm{Hz}$ pure tone with its root mean square (RMS) level identical to the normalized RMS level in speech and noise stimuli was used as the calibration signal during all phases of the experiments. When recording the clear speech sentences all the above criteria were fulfilled via acoustic and digital parameters.

\section{Application of sentences on practice:}

Validation of the sentences were studied via pilot study. The pilot study was done by comparing the perception between conversational and clear speech sentences on five patients having the inclusion criteria of ANSD. The results of the pilot study showed that there were clinically significant differences in perception between the two styles of speech.

\section{Procedure:}

Each participant in the two groups was subjected to the following: Written consent was taken from all participants. All participants were tested in a double-walled, soundtreated room.

The stimulus was presented to the best discriminating ear. The lists were presented at the most comfortable loudness level in both quiet and noise conditions.

To familiarize the participants with the test materials and procedures, a short session with 3 sentences in quiet were conducted at the beginning of the test.

In experiments involving background noise, the sentences were mixed with a speech-spectrum- 
shaped noise at different signal to noise ratios (SNRs) $(+15,+10,+5$ and 0$)$.

All subjects were presented with stimuli via headphone in 6 conditions as follow:

Condition No. I: conversational sentences were presented in quiet and the participant was asked to repeat the sentences.

Condition No. II: clear speech sentences were presented in quiet and the participant was asked to repeat the sentences.

\section{RESULTS}

Table (1): Mean, SD, and range of the 6 conditions in normal subjects:

\begin{tabular}{lllll}
\hline Condition & Mean & SD & Range & $(95 \%$ CI) \\
\hline Condition I (Conversational) & 99.3 & 2.36 & $88-100$ & $98.6<\mu<100$ \\
Condition II (Clear) & 100 & 0 & $100-100$ & $100<\mu<100$ \\
Condition III (Clear SNR +15) & 98.9 & 3.14 & $84-100$ & $97.95<\mu<99.85$ \\
Condition IV (Clear SNR+10) & 97.7 & 7.89 & $60-100$ & $95.32<\mu<100$ \\
Condition V (Clear SNR + 5) & 93.1 & 10.8 & $56-100$ & $89.85<\mu<96.35$ \\
Condition VI (Clear SNR 0) & 43.3 & 20.7 & $8-100$ & $37.06<\mu<49.54$
\end{tabular}

*The results were selected for the right ear in all subjects as there were no differences between both ears.

Table (2): Mean and SD in all conditions in ANSD and SNHL patients:

\begin{tabular}{|c|c|c|c|c|c|c|c|c|}
\hline \multirow[t]{2}{*}{ Condition } & \multicolumn{3}{|c|}{ SNHL } & \multicolumn{3}{|c|}{ ANSD } & \multirow[t]{2}{*}{$\mathrm{T}$} & \multirow[t]{2}{*}{$\mathrm{P}$} \\
\hline & Mean & $\mathrm{SD}$ & Range & Mean & $\mathrm{SE}$ & Range & & \\
\hline Condition I (Conversational) & 92.8 & 8.9 & $52-76$ & 33.1 & 5.77 & $4-60$ & 34.31 & 0.000 \\
\hline Condition II (Clear) & 97.9 & 3.75 & $64-92$ & 49.8 & 5.76 & $20-88$ & 86.23 & 0.000 \\
\hline Condition III (Clear SNR +15) & 92.9 & 10.11 & $60-80$ & 18.7 & 5.91 & $16-72$ & 84.27 & 0.000 \\
\hline Condition IV (Clear SNR + 10) & 78.9 & 19.1 & $56-72$ & 9.3 & 5.86 & $12-60$ & 9.06 & 0.000 \\
\hline Condition V (Clear SNR + 5) & 41.5 & 26.3 & $52-64$ & 2.35 & 4.03 & $12-52$ & 5.44 & 0.000 \\
\hline Condition VI (Clear SNR + 0) & 6.1 & 10.6 & $32-44$ & 0.10 & 3.07 & $4-40$ & 1.83 & 0.044 \\
\hline
\end{tabular}

There was a highly statistically significant differences between ANSD and SNHL patients in conditions I, II, III, IV and V. There was a statistically significant difference between ANSD and SNHL patients in condition VI. 
Grouping of SNHL patients according to the results in condition II (clear speech) into group (A), which had results near normal subjects (score 80-100\%) and group (B), which had results away from normal subjects (score lower than $80 \%$ ):

Table (3): No and \% of the two subgroups of SNHL patients:

\begin{tabular}{lll}
\hline Groups & No & $\%$ \\
\hline Group A & 27 & 90 \\
Group B & 3 & 10 \\
\hline
\end{tabular}

Table (4): Mean, SD and ANOVA study of factors affecting the two subgroups of SNHL patients:

\begin{tabular}{lcccccc}
\hline & & Group A & & Group B & F & P \\
\hline & Mean & SD & Mean & SD & & 0.22 \\
Age & 42 & 10.35 & 34 & 12.33 & 1.58 & 0.73 \\
$\begin{array}{l}\text { Duration of } \\
\text { HL/year }\end{array}$ & 4 & 2.83 & 4.6 & 2.05 & 0.13 & $0.001^{* *}$ \\
Degree of HL & 41.15 & 7.96 & 58.33 & 6.24 & 12.93 & $0.002^{* *}$ \\
WD \% & 86.81 & 6.87 & 72 & 9.8 & 11.68 & $0.000^{* * *}$ \\
Convers. speech & 95.26 & 4.87 & 70.67 & 4.99 & 68.59 & $0.000^{* * *}$ \\
Clear speech & 98.96 & 1.75 & 88 & 0 & 114.05 & \\
\hline
\end{tabular}

*There was a statistically significant difference between the 2 subgroups in the degree of hearing loss, WD \%, conversational speech results and clear speech results.

** means statistical significance

*** means highly significant

Grouping of ANSD patients according to the results in condition II (clear speech) into group (A), which had results near normal subjects (score 80-100 \%) and group (B), which had results away from normal subjects (score lower than $80 \%$ ):

Table (5): No and \% of the two subgroups of ANSD patients:

\begin{tabular}{lll}
\hline Groups & No & $\%$ \\
\hline Group A & 7 & 17.5 \\
Group B & 33 & 82.5 \\
\hline
\end{tabular}

Table (6): Mean, SD and ANOVA study of factors affecting the two subgroups of ANSD patients:

\begin{tabular}{|c|c|c|c|c|c|c|}
\hline & & up A & & oup B & $\mathrm{F}$ & $\mathrm{P}$ \\
\hline & Mean & SD & Mean & SD & & \\
\hline Age & 28 & 4.47 & 26.6 & 8.12 & 0.19 & 0.663 \\
\hline Duration of HL/year & 3.7 & 1.39 & 4.9 & 3.08 & 1.003 & 0.323 \\
\hline Degree of HL & 32.29 & 6.69 & 40.88 & 12.26 & 3.189 & 0.082 \\
\hline WD \% & 54.29 & 24.83 & 19.39 & 21.37 & 14.596 & $0.000^{* * *}$ \\
\hline Conversational speech & 93.39 & 8.20 & 20.36 & 25.19 & 56.518 & $0.000 * * *$ \\
\hline Clear speech & 98.86 & 1.81 & 39.39 & 32.11 & 23.509 & $0.000 * * *$ \\
\hline
\end{tabular}

*There was a highly statistically significant difference between the 2 subgroups in WD \%, conversational speech results and clear speech results. 
Table (7): ANOVA table showing the differences between all conditions in normal, SNHL and ANSD groups:

\begin{tabular}{lccccc}
\hline \multicolumn{1}{c}{ Condition } & Normal & SNHL & AN & F & P \\
\hline Condition I (Conversational) & $99.3+2.36$ & $92.8+8.9$ & $33.1+5.77$ & 85.23 & $0.000^{* * *}$ \\
Condition II (Clear) & $100+0$ & $97.9+3.75$ & $49.8+5.91$ & 51.27 & $0.000^{* * *}$ \\
Condition III (Clear SNR +15) & $98.9+3.14$ & $92.9+10.11$ & $18.7+4.03$ & 239.1 & $0.000^{* * *}$ \\
Condition IV (Clear SNR + 10) & $97.7+7.89$ & $78.9+19.1$ & $9.3+3.07$ & 277.1 & $0.000^{* * *}$ \\
Condition V (Clear SNR + 5) & $93.1+10.8$ & $41.5+26.3$ & $2.35+1.18$ & 267.1 & $0.000^{* * *}$ \\
Condition VI (Clear SNR + 0) & $43.3+20.7$ & $6.1+10.6$ & $0.10+0.10$ & 106.2 & $0.000^{* * *}$ \\
\hline
\end{tabular}

*There was a highly statistically significant difference between the 3 groups in all conditions.

\section{Correlation study in ANSD group:}

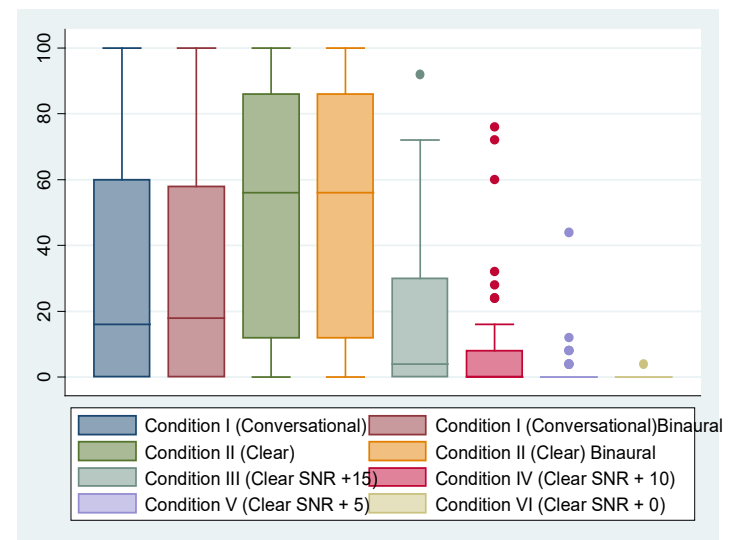

Graph 1: Results of the 6 conditions in AN patients

Correlation studies for ANSD patients described in tables ( 9 and10). There was a strong positive correlation among WD\%, condition I and condition II in ANSD patients. There were highly statistically significant differences among WD\%, condition I and condition II in ANSD patients.

Table (8): Correlation among WD\%, condition I and condition II in ANSD patients:

\begin{tabular}{|c|c|c|c|c|}
\hline & $\mathrm{WD} \%$ & Condition I Monaural & Condition I Binaural & Condition II Monaural \\
\hline \multicolumn{5}{|c|}{ Condition I Monaural } \\
\hline $\mathrm{R}$ & 0.84 & & & \\
\hline$P$ value & $<0.0001 *$ & & & \\
\hline \multicolumn{5}{|c|}{ Condition I Binaural } \\
\hline $\mathrm{R}$ & 0.84 & 0.998 & & \\
\hline$P$ value & $<0.0001^{*}$ & $<0.0001^{*}$ & & \\
\hline \multicolumn{5}{|c|}{ Condition II Monaural } \\
\hline $\mathrm{R}$ & 0.83 & 0.94 & 0.94 & \\
\hline$P$ value & $<0.0001 *$ & $<0.0001^{*}$ & $<0.0001^{*}$ & \\
\hline \multicolumn{5}{|c|}{ Condition II Binaural } \\
\hline $\mathrm{R}$ & 0.83 & 0.94 & 0.94 & 0.997 \\
\hline$P$ value & $<0.0001 *$ & $<0.0001^{*}$ & $<0.0001^{*}$ & $<0.0001^{*}$ \\
\hline
\end{tabular}

* means statistical significance 


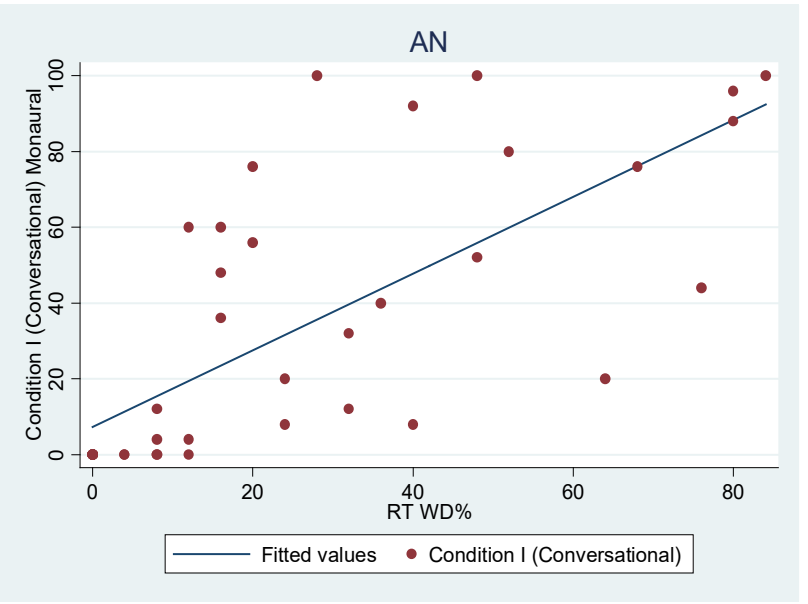

Graph 2: Correlation between WD\% and Condition I (Conversational Monaural) in AN patients.

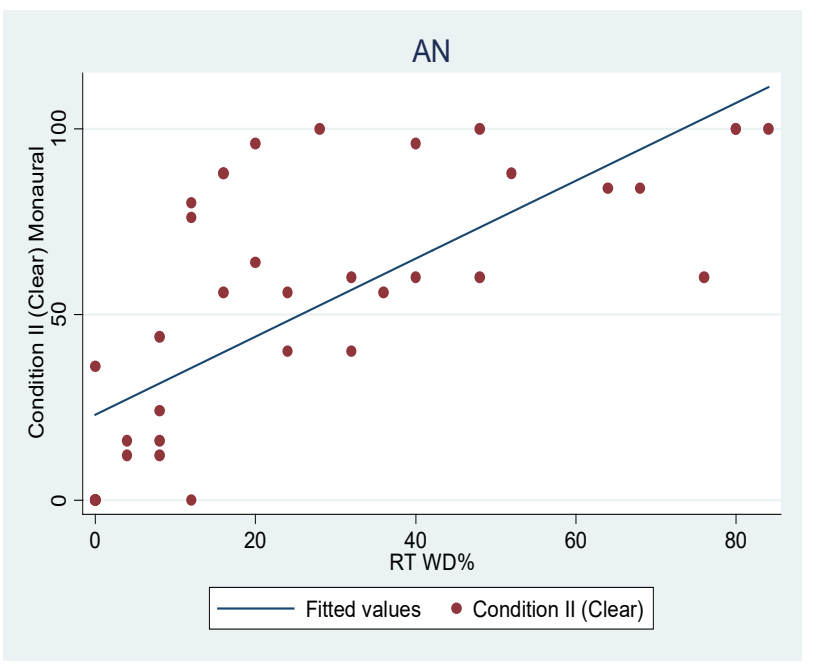

Graph 3: Correlation between WD\% and Condition II (Clear Monaural) in ANSD patients.

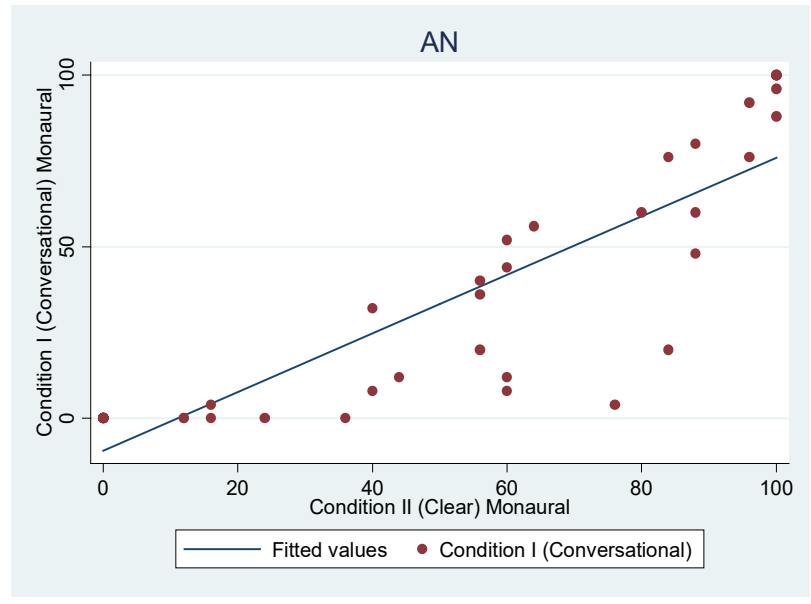

Graph 4: Correlation between Condition I (Conversational Monaural) and Condition II (Clear Monaural) in ANSD patients.

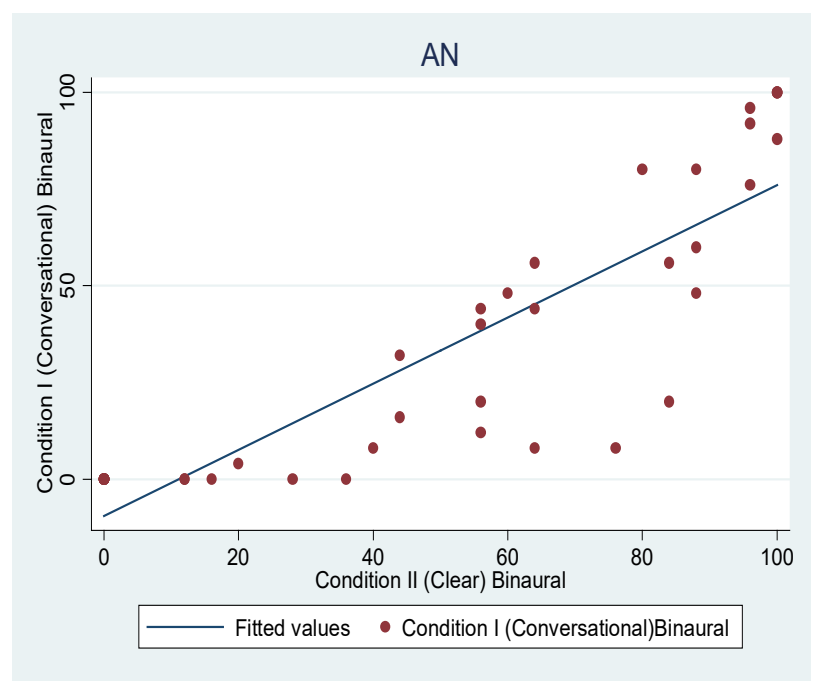

Graph 5: Correlation between Condition I (Conversational Binaural) and Condition II (Clear Binaural) in ANSD patient

Table (9): Correlation among conditions III, IV, V and VI in ANSD patients:

\begin{tabular}{|c|c|c|c|}
\hline & Condition III (Clear SNR +15) & Condition IV (Clear SNR + 10) & Condition V $($ Clear SNR + 5) \\
\hline \multicolumn{4}{|c|}{ Condition IV (Clear SNR + 10) } \\
\hline $\mathrm{R}$ & 0.82 & & \\
\hline$P$ value & $<0.0001 *$ & & \\
\hline \multicolumn{4}{|c|}{ Condition V (Clear SNR + 5} \\
\hline Correlation co-efficient & 0.55 & 0.68 & \\
\hline$P$ value & 0.0004 & $<0.0001 *$ & \\
\hline \multicolumn{4}{|c|}{ Condition VI (Clear SNR + 0) } \\
\hline $\mathrm{R}$ & 0.24 & 0.29 & 0.36 \\
\hline$P$ value & 0.15 & 0.08 & 0.02 \\
\hline
\end{tabular}

* means statistical significance 


\section{DISCUSSION}

The present study included 100 adult subjects 30 of them had normal peripheral hearing, 40 patients with ANSD and 30 had SNHL.

\section{Clear speech tests results:}

\section{Standardization of clear speech:}

To standardize clear speech material, the authors compared perception of conversational versus clear speech in normal hearing subjects. In the present study, the authors used clear speech in slow rate which means that at a longer duration than conversational speech (clear/slow); there was no statistical significant difference between the performance in both conditions in normal subjects as the mean values were 99.3 and 100, respectively though clear speech scores are slightly better than conversational speech (Table 1). These results agreed with the study which was done by Krause and Braida (2002) on normal hearing subjects. Their study showed that clear speech at normal rates, at the same duration of conversational speech, was more intelligible than conversational speech and was almost as beneficial as clear speech at slow rates (clear/slow).

\section{Clear speech results in SNHL patients:}

Evaluation of performance of patients with sensorineural hearing loss (SNHL) on Arabic clear speech showed only $5 \%$ clear speech advantage over conversational speech (Table 2). This agreed with the study which was done by Ferguson (2012) who tried to evaluate the range of talker variability for vowel intelligibility in clear versus conversational speech for older adults with SNHL.The low benefit of clear speech in SNHL patients in the present study may be attributed to the selection of patients as the majority of them had mild and moderate degrees of hearing loss.

SNHL patients are classified into two groups (Table 3). Group A included those who respond to clear speech as normal participants and group B included those who deviate from norms. In group A, $90 \%$ of SNHL patients respond nearly as normal participants to clear speech material. Table 4 showed mean, SD and ANOVA study for the effect of different variables on clear speech in SNHL. The main factors affecting these results are the degree of hearing loss and word discrimination scores. This means better degree of hearing loss and better WD scores for SNHL patients the more likely the patient can benefit from clear speech.

\section{Clear speech results in ANSD patients:}

Performance of ANSD patients on Arabic clear speech demonstrates a significant clear speech advantage in those patients as the mean value of conversational speech is $33.1 \%$ while in clear speech condition is $49.8 \%$ with an advantage equals to $16.7 \%$ for clear over conversational speech (Table 2). This agrees with the study which was done by Zeng et al. (2006). Their study was done on 13 ANSD patients that showed $28 \%$ correct scores of the conversational speech whereas the clear speech produced $44 \%$ correct intelligibility. Another study reported clear speech advantage over conversational speech by Smiljanic and Bradlow, 2009.

In the present study, ANSD patients were classified into two groups. Group A included those who respond to clear speech as normal participants and group B included those who show reduce scores below norms (Table 5). Group A represents only $7 \%$ of ANSD patients which means that majority of ANSD patients deviated away from normal. The results of the present study showed that word discrimination scores is the main factor contributing to clear speech benefit (Table 6).

ANOVA test was used to evaluate the performance of the three groups in Arabic clear speech. It showed highly statistically significant differences between the three groups with reduced performance in AN than normal and SNHL groups in all conditions (Table 7). This difference could be explained by the pathological aspect of ANSD that could be an insult to inner hair cells, spiral ganglion cell disorder, depleted neuronal populations in the auditory brain stem, and demyelination of the auditory nerve. Meanwhile, it was just statistically significant difference in condition VI when SNR was zero as in this condition both ANSD and SNHL patients has poor performance as this represents a challenging condition for both groups.

\section{Correlation study in ANSD:}

Correlation study between conversational and clear speech reveled a strong positive correlation between the two conditions I and II in all participants. As participants who had reduced scores in conversational speech they had similarly reduced scores in clear speech. This means that those who have good conversational scores can benefit more from clear speech.

There was a positive correlation between all conditions involving those with noise administration. This indicates that noise has a drastic effect on 
performance of ANSD patients. These findings are reported by Zeng et al. (2006). However, Liu et al. (2004) showed that noise had no detrimental effect on speech perception at $5 \mathrm{~dB}$ SNR but only decreased speech perception by $15 \%$ at $0 \mathrm{~dB}$ SNR in AN patients.

The physiological and perceptual mechanisms underlying this extreme difficulty remain unclear. Several psychophysical studies have demonstrated poor temporal and spectral processing in participants with ANSD (Rance et al., 2004; Zeng et al., 2005)

In particular, Zeng et al. (2005) found that participants with ANSD exhibited not only 10-20 $\mathrm{dB}$ excessive simultaneous masking for detection of tones in noise, but also had prolonged threshold elevations in both backward and forward masking. At a physiological level, the observed excessive masking may be due to either loss of inner hair cells (also called dead regions in the cochlea) or loss of spike synchrony resulting from damaged nerve fibers (Moser, 2006). This can occur in ANSD when the pathology was mainly affecting IHCs. At a functional level, the excessive masking contributes directly to the extreme difficulty of understanding speech in noise because the perceptual SNR would be much lower than the physical SNR in participants with ANSD. These comparative data, combined with previous results (Starr et al., 2001), are consistent with the subjective complaint by the participants with ANSD of extreme difficulty when listening in noise.

The effects of different factors on the perception of clear speech in ANSD patients were studied. The results showed a statistical significant difference between degree of hearing loss and clear speech perception in ANSD. Age, degree of graduation and duration of hearing loss showed no significant effect on clear speech.

The present study showed that clear speech perception is more intelligible than conversational speech in ANSD and SNHL patients. This improvement is minimal in SNHL patients, while it is marked in ANSD patients. The improvement in perception of clear speech than conversational speech in ANSD patients can be explained by some factors. One of these factors is that clear speech is characterized by greater temporal amplitude modulations than conversational speech. Because a temporal processing deficit is a hallmark of ANSD. The enhanced temporal properties in clear speech may be especially beneficial to individuals with ANSD. Other factor is that clear speech production has the effect of acoustic-phonetic modification of speech signals. Distinctiveness between phonological categories is enhanced in clear speech compared with conversational speech. This study suggested application of clear speech in remediation of patients with ANSD in order to improve their ability to communicate and the use of special type of hearing aids that enhance temporal processing and improve speech perception in noise as an option for management of ANSD.

\section{RECOMMENDATIONS}

Application of clear speech in remediation of patients with ANSD in order to improve their ability to communicate. To use special type of hearing aids, enhances temporal processing and improve speech perception in noise, as an option for management of auditory neuropathy. Also, uses of standardized clear speech material in evaluation of patients complaining of adverse listening conditions e.g. hearing impaired, learning disability and cochlear implant patients.

\section{CONFLICT OF INTEREST}

There are no conflict of interest

\section{REFERENCES}

1. Berlin, C., Li, L., and Hood, L. (2002): Auditory Neuropathy/Dys-Synchrony: After the Diagnosis, then what? Seminars in Hearing, 23, 3: 209-214.

2. Berlin, C. Hood1, L., Morlet, T. Wilensky, D., et al., (2010): Multi-site diagnosis and management of 260 patients with Auditory Neuropathy/Dyssynchrony (Auditory Neuropathy Spectrum Disorder). International Journal of Audiology. 49: 30-43.

3. Beutner, D., Foerst, A., Lang, R., Roth, H. and Walger, M. (2007): Risk factors for auditory neuropathy/auditory synaptopathy. Otorhinolaryngol, 69: 239-244.

4. Bielecki I, Horbulewicz A, Wolan T. (2012): Prevalence and risk factors for auditory neuropathy spectrum disorder in a screened newborn population at risk for hearing loss. Int J Pediatr Otorhinolaryngol 76: $1668-70$.

5. Ferguson, S. (2012): Vowel intelligibility in clear and conversational speech for normal-hearing and hearingimpaired listeners. Journal of the Acoustical Society of America, 112: 259-271.

6. Gunay, K., Bulent, S., Taner, K. and Kerim, C. (2008): The frequency of auditory neuropathy detected by universal newborn hearing screening program. International Journal of Pediatric Otorhinolaryngology, 72: 1461-1469. 
7. Hassan, D. (2008): Auditory Neuropathy: An approach to management. Reprint from the scientific Journal of Al-Azhar Medical Faculity \{Girls\} 29: (3) 1533-1544.

8. Hood, L. Berlin, C. Morlet, T. Brashears, S. Rose, K. and Tedesco, S. (2002): Considerations in the clinical evaluation of auditory neuropathy/auditory dys-synchrony. Seminar of Hearing, 23: (3)201-208.

9. Hood, L., Berlin, C., Bordelon, J., and Rose, K. (2003): Patients with auditory neuropathy/dys-synchrony lack efferent suppression of transient evoked otoacoustic emissions. Journal of the American Academy of Audiology, 14: 302-313.

10. Krause, J. and Braida, L. (2002): Investigating alternative forms of clear speech: The effects of speaking rate and speaking mode on intelligibility. Journal of the Acoustical Society of America, 112: 2165-2172.

11. Liu, S., Del Rio, E., Bradlow, A. and Zeng, F. (2004): Clear speech perception in acoustic and electric hearing. Journal of the Acoustical Society of America, 116: 2374-2383.

12. Moser, T. (2006): Diagnosis and therapy of auditory synaptopathy/neuropathy. HNO 54: 833-839 (article in German).

13. Nikolopoulos TP. (2014): Auditory dyssynchrony or auditory neuropathy: understanding the pathophysiology and exploring methods of treatment. Int J Pediatr Otorhinolaryngol 78: 171-3.

14. Petros, V., Thomas, P., Nikolopoulos, B., Evangelia, T., George, P., and Stavros, K. (2008): Auditory neuropathy: Endocochlear lesion or temporal processing impairment? Implications for diagnosis and management. International Journal of Pediatric Otorhinolaryngology, 72: 1135-1150.

15. Rance, G., McKay, C. and Grayden, D. (2004): Perceptual Characterization of Children with Auditory Neuropathy. Ear and Hearing 25:34-46.
16. Rapin, I. and Gravel, J. (2003). "Auditory neuropathy": physiologic and pathologic evidence calls for more diagnostic specificity. International Journal Pediatric Otorhinolaryngol, 67: 707-728.

17. Rosamaria, S., Arnold, S., Henry, J., Michalewski, B and Edoardo, A. (2008): Neural and receptor cochlear potentials obtained by transtympanic electrocochleography in auditory neuropathy. Clinical Neurophysiology, 119: 1028-1041.

18. Simmons, J. and McCreery, R. (2007) Auditory Neuropathy/Dys-synchrony: Trends in Assessment and Treatment. The ASHA Leader, 12(8), 12-15.

19. Starr, A., Picton, T., Sininger, S., Hood, L. and Berlin, I. (1996): Auditory Neuropathy. Brain, 119:741-753.

20. Starr, A., Sininger, Y., Nguyen, Y., Michalewski, H., Oba, S., Abdala,C. (2001): Cochlear receptor (microphonic and summating potentials, otoacoustic emissions) and auditory pathway (auditory brain stem potentials) activity in auditory neuropathy. Ear Hear. 22: 91-99.

21. Smiljanic, R., Bradlow, A. (2008): "Stability of temporal contrasts across speaking styles in English and Croatian”. J. Phonetics 36: 91-113.

22. Soliman, S. (1987): low frequency syndrome (auditory neuropathy): further study, paper presented at the 1998 Egyptian ORL Society Conference, Cairo, Egypt.

23. Spirakis S (2012) Auditory Neuropathy Spectrum Disorder and Hearing Aids: Rethinking Fitting Strategies Hearing Review 18: 28-33.

24. Zeng, F., Kong, Y., Michalewski, H. and Starr, A. (2005): Perceptual consequences of disrupted auditory nerve activity. Journal of Neurophysiology, 93: 3050-3063.

25. Zeng, F., Liu, S. (2006): Speech perception in individuals with auditory neuropathy, J. Speech Lang. Hear. Res. 49: 367-380. 